\title{
Contribution à l'étude faunistique et biogéographique des Gastéropodes du secteur roumain du Danube
}

\author{
A. Negrea 1
}

Mots clés : Gastropoda, faunistique, biogéographie, Danube, Roumanie.

La première synthèse sur les Gastéropodes signalés à ce jour dans le secteur roumain du Danube (chenal, systèmes lacustres de la zone inondable, delta) avec mise à jour taxonomique (classification, synonymies, corrections) est présentée. Pour chacune des 54 espèces recensées sont indiqués : l'aire de répartition sur le globe et dans le bassin du Danube, une brève caractérisation écologique, le matériel étudié par l'auteur et les données de la bibliographie sélectionnées d'une manière critique. Le travail s'achève par l'analyse biogéographique des espèces étudiées. Celle-ci souligne le nombre relativement élevé des espèces endémiques à aire de répartition restreinte.

\section{A faunistic and biogeographical study of the Gastropoda of the Roumanian sector of the Danube}

Keywords : Gastropoda, faunistic, biogeography, Danube, Roumania.

This is the first synthesis of the Gastropoda reported up to the present in the Roumanian sector of the Danube (main channel, lake systems of the flooded zone, delta) together with an up to date summary of their taxonomy (classification, synonomies, corrections). The following information is provided for each of the 54 species recorded : the area of their distribution globally and in the Danube basin, a brief summary of their ecological characteristics, the material studied by the author and data critically selected from the bibliography. The work ends with a biogeographical analysis of the species studied. This emphasizes the relatively high number of endemic species with a limited distribution.

\section{Introduction}

Les Gastéropodes jouent un rôle important dans l'économie des eaux du Danube, y compris dans celles de la zone inondable et de son delta.

L'effectif de certaines espèces constitue une partie de la nourriture des poissons qui consomment leurs pontes, larves et adultes. On connaît, également, l'intérêt de quelques espèces en tant qu'indicateurs biologiques de la qualité de l'eau.

Etant donné l'importance de ces organismes, le présent travail se propose d'établir une synthèse des données faunistiques et biogéographiques concernant les espèces recensées à ce jour dans le secteur roumain du Danube, depuis Bazias jusqu'à son embouchure dans la Mer Noire (Fig. 1). Dans ce but nous avons utilisé à la fois des données personnelles inédites, et celles de la littérature. Il s'agit principalement des travaux de Bacescu (1948), Busnita

\footnotetext{
- 1. Institut de Spéologie « Emile Racovitza », str. Frumoasa 11, 78114 Bucarest, Roumanie.
}

et al. (1970), Cure et al. (1974-1975), Enaceanu (1967), Enaceanu et al. (1964), Frank et al. (1990), Grossu (1956, 1963, 1986, 1987, 1993), Grossu et al. (1956, 1968, 1975), Jaeckel (1967), Markovski (1955), Negrea et al. (1975, 1992), Popescu (1963), Popescu et al. $(1961,1962)$, Popescu-Gorj et al. (1956, 1961), Popescu-Marinescu (1973, 1992), Popescu-Marinescu et al. (1980), Prunescu-Arion et al. (1965), Russev (1967), Starobogatov (1970), Zilch et al. (1962). Toutes les données de ces travaux ont été rigoureusement vérifiées, en tenant compte des synonymies anciennes et récentes et de la classification utilisée par Grossu (1986-1987) dans son importante monographie.

Ce travail représente la première synthèse concernant les Gastéropodes du secteur roumain du Danube. La publication de Frank et al. (1990), contient des données approfondies et à jour pour les secteurs allemand, autrichien, tchécoslovaque et hongrois du Danube, mais les données concernant le secteur roumain, extraites en majorité de Grossu \& Grossu (1968), couvrent un faible nombre de stations. 


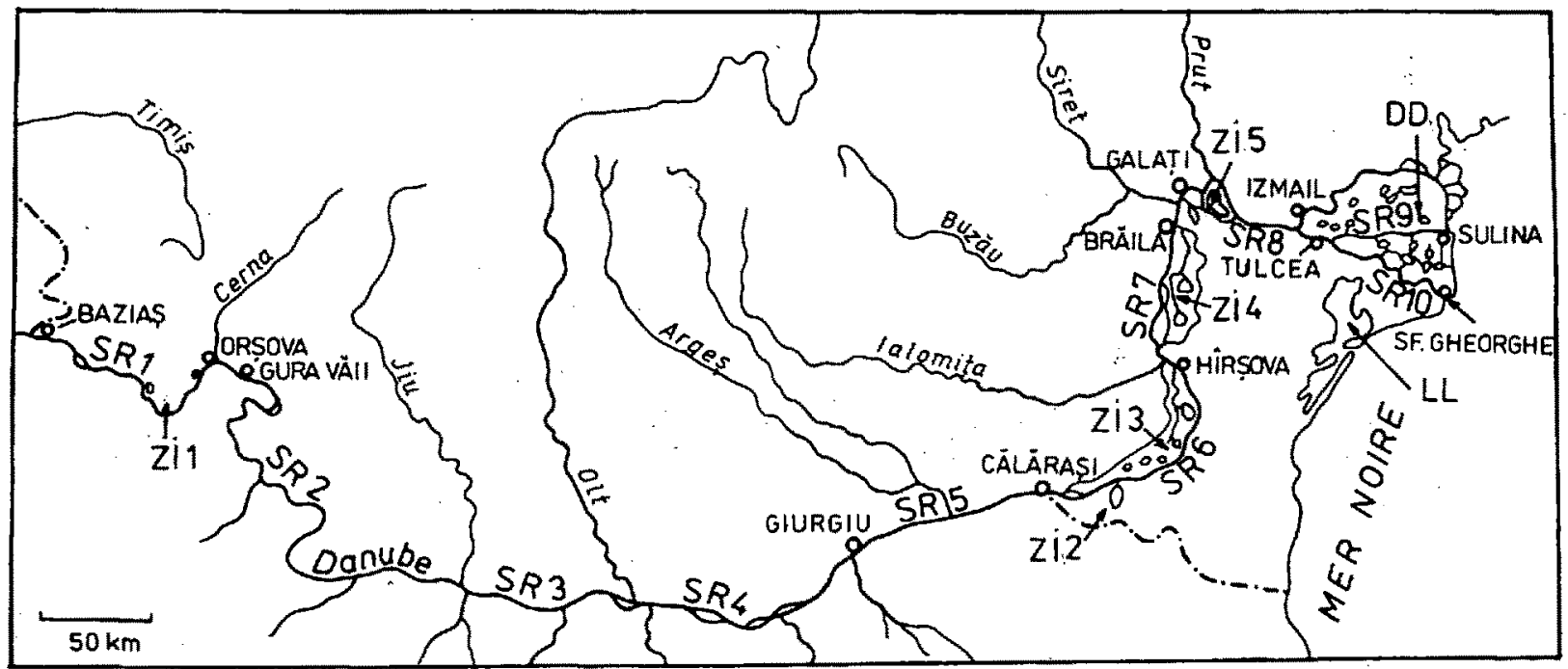

Fig. 1. Secteur roumain du Danube. SR = secteur roumain du Danube, $\mathrm{km}$ 1055-943 (Bazias - Sulina) : SR $1=\mathrm{km} 1055-943$ (Bazias

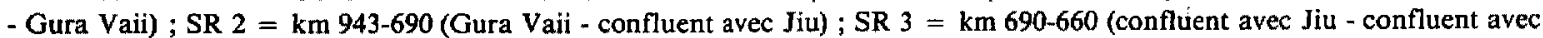

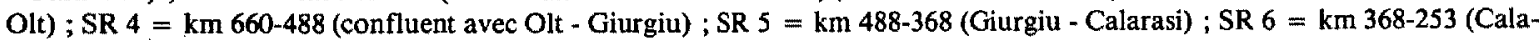

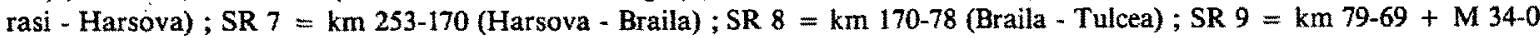

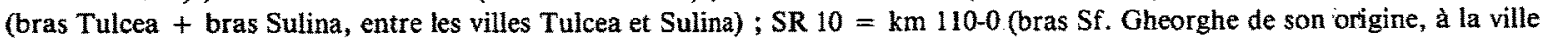
Sf. Gheorghe). ZI = zone inondable du Danube : ZI 1 = petits lacs dans le défilé du Danube entre Bazias et Gura Vaii ; ZI 2 = lac Oltina ; ZI 3 = lacs et canaux de l'lle Borcea; ZI 4 = lacs et canaux de la "Grande lle » et de la "Petite ile » de Braila ; ZI 5 = lacs et canaux du système Crapina-Jijila. DD = lacs et canaux du Delta du Danube. LL = lacs littoraux de la mer Noire près du Delta du Danube (complexe des lacs Razim).

Fig. 1. Roumanian sector of the Danube. $\mathrm{SR}=$ Roumanian sector of the Danube, $\mathrm{km} 1055-943$ (Bazias - Sulina) : SR $1=\mathrm{km}$

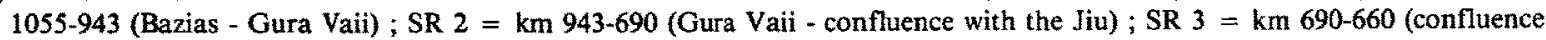
with the Jiu - confluence with the Olt) ; SR $4=\mathrm{km} 660-488$ (confluence with the Olt - Giurgiu) ; SR $5=\mathrm{km} 488-368$ (Giurgiu - Calarasi); SR $6=\mathrm{km} \mathrm{368-253} \mathrm{(Calarasi} \mathrm{-} \mathrm{Harsova);} \mathrm{SR} 7=\mathrm{km} \mathrm{253-170} \mathrm{(Harsova} \mathrm{-} \mathrm{Braila);} \mathrm{SR} 8=\mathrm{km} \mathrm{170-78} \mathrm{(Braila}$ - Tulcea) ; SR $9=\mathrm{km} \mathrm{79-69+M} \mathrm{34-0} \mathrm{(Tulcea} \mathrm{arm} \mathrm{+} \mathrm{Sulina} \mathrm{arm} \mathrm{between} \mathrm{the} \mathrm{towns} \mathrm{Tulcea} \mathrm{and} \mathrm{Sulina)} \mathrm{;} \mathrm{SR} 10=\mathrm{km} 110-0$ (arm Sf. Gheorghe from its origin, to the town Sf. Gheorghe). ZI = flooding zone of the Danube : ZI $1=$ small lakes in the ravine of the Danube between Bazias and Gura Vaii ; ZI 2 = lake Oltina ; ZI 3 = lakes and canals of l'ile Borcea; ZI $4=$ lakes and canals of the « Grande Ile » and the « Petite île » of Braila ; ZI 5 = lakes and canals of the system Crapina-Jijila. $\mathrm{DD}=$ lakes and canals of the Danube Delta. $\mathrm{LL}=$ coastal lakes of the Black Sea near the Danube Delta (complex of the lakes of Razim).

Un autre travail relatif au même groupe d'organismes est celui, édité en roumain par Negrea \& Popescu-Marinescu (1992). Toutefois ce dernier s'inscrit dans une autre perspective. Consacré à la dynamique quantitative des populations de Gastéropodes (fréquence, densité numérique, biomasse, conditions de milieu), ce travail néglige nombre de stations et s'intéresse seulement aux espèces écologiquement importantes.

\section{Matériel et méthodes}

La méthode d'investigation pour dresser l'inventaire des Gastéropodes du secteur roumain du Danube est essentiellement qualitative :
- draguage du Danube entre Bazias et les diverses embouchures dans la mer Noire (Fig. 1 : SR 1-10), des lacs et des canaux de la zone inondable (Fig. 1 : ZI 1-5), du delta, y compris le golfe Musura de la mer Noire (nord de Sulina) et l'île Sacalin (sud de Sf. Gheorghe) (Fig. 1 : DD) ;

- tri des coquilles (avec ou sans animal) de chaque échantillon et étude à la loupe binoculaire.

Des échantillons quantitatifs ont cependant été effectués simultanément dans lé système de lacs et canaux Crapina-Jijila de la zone inondable en 19561957 et 1966-1967, fournissant des données utilisées dans Negrea \& Negrea (1975) et Negrea \& PopescụMarinescu (1992). Dans cette même zone, de 
nombreux échantillons qualitatifs étudiẹ́s dans ce travail, ont été réalisés de 1956 à 1992.

Enfin, en complément de cette étude, la littérature se rapportant au secteur roumain du Danube a été soigneusement analysée, comme noté ci-avant dans l'introduction.

Les résultats sont présentés dans le chapitre suivant et synthétisés dans le table $\mathrm{I}$.

Pour chacune des 54 espèces recensées sont indiquées : l'aire de répartition sur le globe et dans le bassin du Danube, une brève caractérisation écologique (affinités écologiques pour les eaux courantes ou stagnantes et pour les différents substrats), le matériel étudié par nous-même et les données de la bibliographie.

Toutes les stations sont mises en ordre géographique d'amont en aval (Fig. 1) de la manière suivante :

- secteur roumain du Danube (SR) divisé en dix secteurs (SR 1 - SR 10) ;

- zone inondable du Danube $(\mathrm{ZI})$ divisée en cinq systèmes lacustres (ZI 1 - ZI 5) ;

- delta du Danube (DD) ;

- lacs littoraux de la mer Noire : complexe des lacs de Razimé, près du délta du Danube (LL).

Abréviations utilisées dans le texte pour « legit » : $\mathrm{AE}=$ Arion E., CV = Cure V., NA = Negrea A., PM = Papadopol M., PMV = Popescu-Marinescu $V_{.}, S C=$ Stamate C., TL $=$ Talau L., ZV $=$ Zinevici $\mathrm{V}$.

Abréviations pour la bibliographie : $\mathrm{B}=$ Bacescu M. (1948), $E$ = Enaceanu V. \& Brezeanu Gh. (1964), F = Frank C., Jungbluth J. \& Richnovsky A. (1990), G 1 = Grossu A.V. (1956), G 2 = Grossu A.V. (1963), G 3 = Grossu A.V. (1986), G $4=$ Grossu A.V. (1987), G 5 = Grossu A.V. \& Grossu D. (1968), G 6 = Grossu A.V. \& Negrea A. (1975), G 7 = Grossu A.V. \& Paladian G. (1956), MK = Markovski I.M. (1955), N = Negrea A. \& PopescuMarinescu V. (1992), P 1 = Popescu L. \& Munteanu I (1962), P 2 = Popescu V. (1963), P $3=$ Popescu-Gorj A. \& Costea E. (1961), P $4=$ Popescu-Gorj A., Popescu E. \& Georgescu I. (1956), P 5 = Prunescu-Arion E., Elian-Talau L. \& Baltac M. (1965).

\section{Gastéropodes signalés à ce jour dans le secteur roumain du Danube}

\subsection{Neritoidea Marton \& Yonge 1964}

\subsubsection{Neritidae Rafinesque 1815}

Theodoxus (Theodoxus) transversalis (C. Pfeiffer 1828)

Pontique - danubienne. Tout le cours du Danube y compris quelques affluents et le bassin du Dniester).

Eaux courantes, lacs; lithophile et parfois psammo-et pélophile.

Matériel étudié

Danube : SR 1 : confluent avec Cerna, 30.9.1975 (ZV), 3.10.1989 (NA) ; SR 2 : Drobeta-Turnu Severin, 25.3.1972 (CV) : SR 6 : Hârsova, 29.6.1962 (PMV); SR 9 : bras Tulcea à Tulcea, 16.9.1992 (NA), bras Sulina à Crisan, 18.3.1961 et 14.5.1968 (PMV), 19.9.1992 (NA).

Zone inondable du Danube: ZI 5 : lac Crapina, 28.7.1957 (NA).

Lacs littoraux de la Mer Noire: Sinoe à Istria, 10.10.1961 (PMV), 16.8.1968 (NA).

Bibliographie

Danube : SR 1 : Pescari, km 1005 et Mraconia (G5), Svinita et Cazane (B) ; SR 3 : Corabia (N) ; SR 4 : Giurgiu (G5) ; SR 6 : Jirlau-Borcea (G5) et Hârsova (N) ; SR 9: bras Sulina à Maliuc, M 24, Gorgova, M 19, M 2, Sulina (G5), et Crisan (F). Bras Chilia (MK).

Theodoxus (Theodoxus) danubialis (C. Pfeiffer 1828)

Pontique - danubienne tout le cours du Danube et son bassin, Dniester, Bourg et Dniepr).

Eaux courantes, lacs; lithophile et parfois psammo et pélophile.

Matériel étudié

Danube : SR 1 : Moldova Veche, 22.4.1975, 3.7.1975 et 2.10.1975 (ZV), 18.9.1993 (NA), Mraconia, 30.9.1975 (ZV) 25.6.1982 (PMV), Bahna, 23.4.1975 (ZV) ; SR 9 : bras Sulina à Sulina, 4.4.1966, 25.9.1967, 1.6.1974, 11.10.1974 (PMV) et à l'embouchure, 19.9.1992 (NA); SR 10 : Ceatal Sf. Gheorghe, 12.12.1981, (PMV), bras Sf. Gheorghe à $\mathrm{km} \mathrm{7,11.7.1966} \mathrm{(PMV),} \mathrm{à} \mathrm{km} \mathrm{5,}$ 16.4.1967, 4.8.1972 et 29.5.1974 (PMV), à $\mathrm{km} \mathrm{3,}$ 27.7.1967, 5.3.1968 et 25.5.1974 (PMV) et à Sfântu Gheorghe, 4.6.1980 et 20.9.1992 (NA).

Zone inondable du Danube : ZI 3 : canal Saltava, 10.10.1960 (PMV) ; ZI 5 : les lacs Crapina, 15.5.19567.9.1957, 8.6.1966-15.5.1967, 11.7.1990 (NA), Ascunsu, 30.8.1956, 29.5.1957 (NA), Jijila, 18.5.1956-18.4.1958, 9.9.1966 (NA), Combra, 18.5.1956 et Podu, 9.9.1966 (NA), les canaux Latimile, 1.11.1956, 9.9.1957 (NA), Gradinile, 1.5.1957 (NA) et Ciulinet, 1.12.1956 (NA). 
Tableau 1. Gastéropodes du secteur roumain du Danube (SR), de la zone inondable (ZI), du delta du Danube (DD) et des lacs littoraux de la Mer Noire (LL).

Table 1. Gastropods from the Roumanian sector of the Danube (SR), from the flooding zone of the Danube (ZI), from the Danube delta (DD) and from the coastal lakes of the Black Sea.

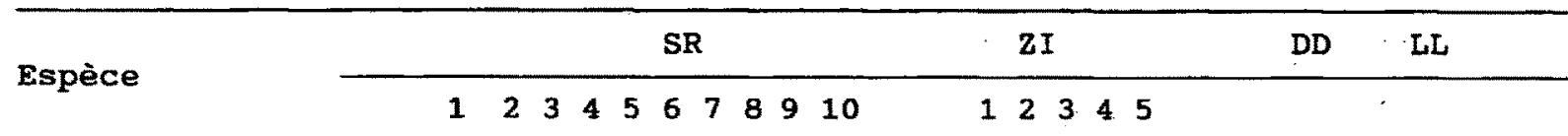

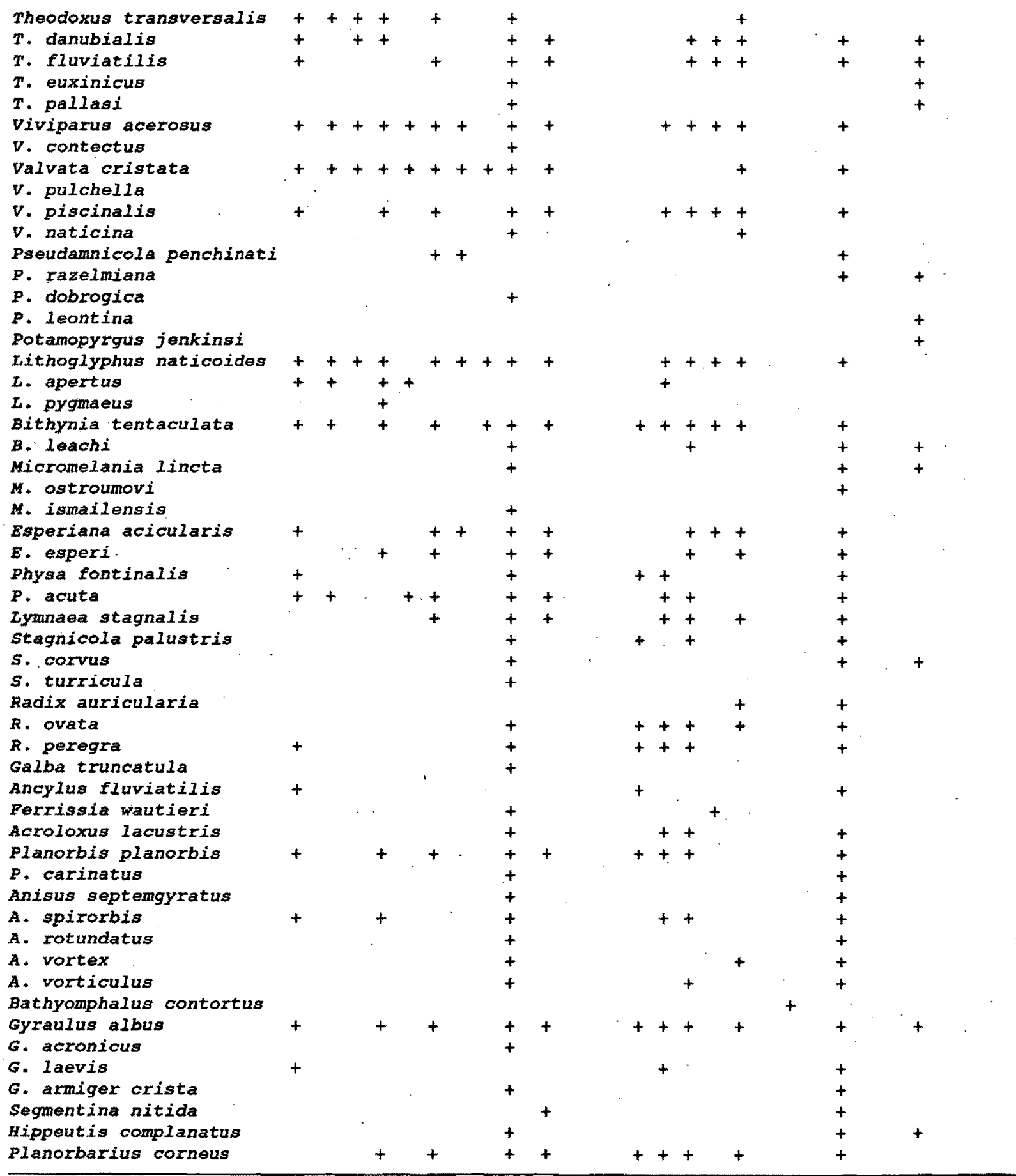


Delta du Danube : lac Trei Iezere, 8.6.1972 (PM), canal Cherhana Musura, 2.10.1967 (PMV), golfe Musura, 4.6.1975 (TL), canal Turetkaia, 18.5.1967, 4.6.1980 (PMV), ile Sacalin, 19.5.1977-14.10.1977 (AE), 28.7.1979 et 9.4.1980-8.10.1980 (PMV).

\section{Bibliographie}

Danube : SR 1 : Pescari et Orsova (G5) ; SR 2 : lac de barrage Portile de Fier II (N) ; SR 3 ; confluent avec l'Olt (N) ; SR 7 : bras Macin (N) ; SR 9 : bras Sulina à Maliuc, M 24, Gorgova, M 19, M 14, M 12, M 9, M 3, M 2 et Sulina (GS) ; SR 10 : Ceatal Sf. Gheorghe (N), bras Sf. Gheorghe à km 0 (G5); bras Chilia (MK).

Zone inondable du Danube : ZI 3 : canal Saltava (P5) ; ZI 4 : île Braila (P5).

Delta du Danube : lac Gorgova (N).

Lacs littoraux de la Mer Noire : Razim à Sarichioi (GI).

\section{Theodoxus (Theodoxus) fluviatilis (Linnaeus 1758)}

Européenne, caractéristique pour les provinces baltique, pontique et danubienne (dans le Danube et son bassin, de la Hongrie jusqu'à l'embouchure ; le Dniepr et le Don).

Eaux à fort courant, mais aussi lacs ; lithophile et parfois psammophile ou même pélophile.

\section{Matériel étudié}

Danube : SR 9 : bras Sulina à Maliuc, 14.5.1968 (PMV) et 16.9.1992 (NA).

Zone inondable du Danube : ZI 5 : lacs Crapina, 20.5.1956-3.8.1957, 6.6.1966-13.5.1967, 11.7.1990 (NA), Ascunsu 30.8.1956 (NA), Popina, 9.6.1966, 8.9.1966 (NA), Cacova, 11.11.1957 (NA), Jijila, 16.5.195617.4.1958 (NA) et Somovelu, 8.2.1958 (NA), canal Gradinile, 8.6.1966-11.5.1967 (NA).

Delta du Danube : lac Furtuna, 12.5.1967 et 16.9.1967 (ZV), canal Cardon, 28.4.1967 (PMV), canal Cherhana Musura, 2.10.1967 (PMV), golfe Musura, 15:7.1975 (TL), île Sacalin, 27.7.1967, 18.9.1975 et 1.11.1975 (PMV).

\section{Bibliographie}

Danube : SR 1 : Svinita (N) ; SR 6 : Jirlau-Borcea (G5) ; SR 9 : Tulcea (N) ; SR 9 : bras Sulina à Maliuc, M 24, M 14, M 12, M 9, M 3, M 2 et à Sulina (G5) ; SR 10 : Ceatal Sf. Gheorghe et bras Sf. Gheorghe a $\mathrm{km} 5$ (G5).

Zone inondable du Danube : ZI 3 : île Borcea $(\mathrm{N})$; ZI 4 : île Braila $(\mathrm{N})$.

Lacs littoraux de la Mer Noire : Razim à Cap Dolos$\operatorname{man}(G 5)$.

\section{Theodoxus (Theodoxus) euxinus (Clessin 1885)}

Endémique dans la province pontique (embouchures du Danube et lac Razim seulement).

Eaux salmastricoles de la zone de contact eaux douceseaux marines (4-5 \% $\mathrm{ClNa}$ ); lithophile, mais aussi psammo- et pélophile (Grossu, 1986).
Matériel étudié

Danube : SR 9 : embouchure du bras Sulina, 16.9.1992 (NA).

\section{Bibliographie}

Danube : SR 9 : embouchure du bras. Sulina (G3).

Lacs littoraux de la Mer Noire : Razim (G3).

Theodoxus (Theodoxus) pallasi (Lindholm 1924)

Espèce pontique-caspienne endémique (M. Noire, $M$. d'Azof et M. Caspienne). Danube : embouchures.

Eaux salmastricoles et parfois dulcicoles; lithophile, mais aussi psammo- et pélophile.

Matériel étudié

Danube : SR 9 : embouchure du Danube à Sulina, 17.9.1992 (NA).

Bibliographie

Danube : SR 9 : embouchure du Danube à Sulina (G3). Lacs littoraux de la Mer Noire : Razim (G3).

\subsection{Mesogastropoda Thiele 1925}

\subsubsection{Viviparidae Gray 1848}

Viviparus (Viviparus) acerosus Bourguignat 1862

Syn. V. danubialis penchinati (Bourg. 1862) sensu Frank et al. 1990.

Pontique-danubienne. Tout le cours du Danube, surtout dans son bassin inférieur.

Eaux stagnantes et à courant lent, bras morts etc ; lithopsammophile (dans le Danube) et pélo-macrophytophile (dans la zone inondable du Danube).

\section{Matériel étudié}

Danube : SR 10 : bras Sf. Gheorghe à km 5, 16.4.1967, 20.5.1974 (PMV), à km 3, 5.3.1968, 20.5.1974 (PMV) et à km 0, 24.7.1971 (PMV).

Zone inondable du Danube : ZI 3 : canal Saltava, 10.10.1960 et 16.4.1962 (PMV); ZI 4 : canál Fílipoiu, 30.6.1962 (PMV); ZI 5: les lacs Crapina, 14.5.1956-8.9.1957, 7.6.1966-12.5.1967 et 11.7.1990 (NA), Ascunsu, 30.8.1956 et 29.5.1957 (NA), Popina, 9.6.1966, 7.11.1966 et 15.5.1967 (NA), Jijila, 19.5.1956-17.4.1958 et 8.9.1966 (NA), Combra, 3.10.1956, 4.5.1957 et 7.2.1958 (NA), Somovelu, 8.2.1958 (NA) et Podu, 9.9.1966 et 12.5.1967 (NA), les canaux Latimile, 1.11.1956 et 9.9.1957 (NA), Gradinile, 1.3.1957 (NA) et Ciulinet, 1.12.1956 (NA).

Delta du Danube : lac Furtuna, 8.5.1967 et 16.9.1967 (ZV), 16.9.1982 (NA), lac Trei Iezere, 8.6.1972 et 4.11.1972 (PM), canal Turetkaia, 17.3.1968, 29.7.1979 et 3.10.1979 (PMV), île Sacalin, 27.7.1967, 10.8.1968 et 18.8.1976 (PMV). 


\section{Bibliographie}

Danube : SR 1 : défilé du Danube (N) ; SR 2 : lac Portile de Fier II (N) ; SR 3 : Potelu (G5) ; SR 4 : Giurgiu (G5) ; SR 5 : Calarasi (G5) ; SR 6 : Jirlau-Borcea (G5) ; SR 7 : bras Macin (G5) ; SR 9 : bras Sulina à Maliuc, $M$ 24 et M 22 (G5), Crisan (F) et bras Dunarea Veche (P2) ; SR 10 : bras Sf. Gheorghe à $\mathrm{km} 5$ (G5).

Zone inondable du Danube : ZI 2 : lac Oltina (G5, P3).

Delta du Danube,: lacs Matita et Merhei $(\mathrm{N})$ et canal Turcu (N).

\section{Viviparus (Viviparus) contectus (Millet 1813)}

Europe (sauf l'extrémité nordique). Tout le cours du Danube.

Eaux stagnantes (lacs, étangs, marais, bras morts) et courantes (rivières, fleuves) ; pélo- et macrophytophile.

\section{Bibliographie}

Danube : SR 9 : bras Sulina à Crisan (F).

\subsubsection{Valvatidae Thomson 1840}

\section{Valvata (Valvata) cristata (O.F. Müller 1774)}

Paléarctique (euro-sibérienne jusqu'à l'Amour). Tout le cours du Danube.

Eaux temporaires, lacs, bras morts, sources, et eaux à courant lent ; pélo- et macrophytophile.

Matériel étudié

Danube : SR 9 : bras Sulina à Maliuc, 13.5.1967 et 11.6.1967 (ZV).

Zone inondable du Danube - ZI 5 : lac Jijila, 3.10.1956, 17.4.1957 et 13.6.1957 (NA).

Delta du Danube :, zone Carasuhat : 10.6.1967, 17.9.1967 ( $\mathrm{ZV}$ ) et lac Iacob : 16.8.1974, 18.10.1972(TL).

\section{Bibliographie}

Danube : SR 1 - SR 9 : chenal, de Pescari à Sulina (G2) ; SR 9 : bras Sulina à Maliuc (P2) et à Crisan (F) ; SR 10 : bras Sf. Gheorghe (G7):

Delta du Danube : lacs Furtuna, Raducu et Stambulet (N) ; également dans la zone inondable et le delta de Chilia (MK).

\section{Valvata (Atropidina) pulchella Studer 1820}

Paléarctique (euro-sibérienne et Libye). Tout le Danube. Eaux périodiques, lacs, fleuves, bras morts ; pélophile.

\section{Bibliographie}

Danube : bras Chilia et les bras du delta Chilia (MK).

Valvata (Cincina) piscinalis (O.F. Müller 1774)

Paléarctique (introduite en Amérique du Nord). Tout le cours du. Danube et ses àffluents.

Eaux à courant lent, lacs, eaux temporaires, bras morts ; pélo-, psammo- et macrophytophile.
Matériel étudié

Danube : ST 1 : Mraconia, 27.7.1973, 9.7.1974 et 23.4.1975 (ZV), 7.5.1981, 28.7.1981, 27.10.1981 et 18.8.1982 (PMV), 22.9.1989 (NA), Cerna, 16.5.1973, 11.4.1974, 9.7.1974, 23.4.1975 et $\cdot 30.9 .1975(\mathrm{ZV})$, 5.10.1991 (NA), Bahna, 6.2.1973, 27.7.1973, 23.4.1975 (ZV) ; SR 9 : bras Sulina à M 2, 2.8.1971 et à M O, 1.6.1974 (PMV); SR 10 : bras Sf. Gheorghe à M 1, 8.7.1979, 4.1.1980, 4.6.1980 et 5.8.1981 (PMV).

Zone inondable du Danube : ZI 3 : canal Saltava, 10.10.1960 (PMV); ZI 4 : canal Filipoiu, 30.6.1962 (PMV) ; ZI 5 : les lacs Crapina, 15.5.1956-7.9.1957, 8.6.1966-13.5.1967 et 11.7.1990 (NA), Ascunsu, 30.8.1956 et 29.5.1957 (NA), Cacova, 11.11.1957 (NA), Jijila, 18.5.1956-18.4.1958 (NA), Combra, 18.5.1956, 4.5.1957 et 7.2.1958 (NA) et Popina 9.6.1966, 7.11.1966 et 15.5.1967 (NA), les canaux Latimile, 1.11.1956 et 9.9.1957 (NA), Ciulinet, 1.12.1956 (NA) et Gradinile, 8.6.1966-11.5.1967 (NA).

Delta du Danube : Les lacs Trei Iezere, 8.6.1972 et 4.11.1972 (PM), Matita, 29.10.1972 (PM) et Iacob, 16.8.1974 (TL), les canaux Cardon, 28.4.1967 (PMV), Imputita, 21.10.1965 (PMV), Gafon, 14.8.1967 (ZV) et Turetkaia, 9.4.1980 et 6.8:1980 (PMV), île Sacalin, $27.7 .1967,7.10 .1968,4.4 .1970,9.8 .1974,3.10 .1979$ et 4.4.1980 (PMV).

Bibliographie

Danube : SR 1 : Pescari et Orsova (G5) ; SR 4 : Giurgiu (G5) ; SR 6 : Dervent (G5) ; SR 9 : bras Sulina à Maliuc, M 24, M 13 M3, M 2 (G5) et à Crisan (F) ; SR 10 : Ceatal Sf. Gheorghe (G5).

Zone inondable du Danube : ZI 2 : lac Oltina (P3) ; ZI 3 : canal Saltava (P5) et Jirlau-Borcea (G5).

Delta du Danube : Les lacs Matita, Merhei, Gorgova, Iacob, Uzlina et Rosu (G7), Furtuna, Puiu, Rosulet, Raducu et Stambulet (N), zone Pardina (P4), les canaux Cardon et Imputita (P2).

\section{Valvata (Borysthenia) naticina Menke 1845}

Espèce pontique-danubienne-baltique. Tout le cours du Danube, le Dniepr et le Don.

Fleuves et leur système lacustre. Litho-rhéophile et pélopsammophile.

\section{Matériel étudié}

Danube : SR 9 : bras Sulina à l'embouchure, 17.9.1992 (NA).

Zone inondable du Danube : ZI 5 : les lacs Crapina, 15.5.1956-11.11.1957, 8.6.1966 et 24.9.1989 (NA), Ascunsu, 30.8.1956 et 29.5.1957 (NA), Jijila, 18.5.1956-7.11.1957, 8.4.1958 (NA) et Combra; 18.5.1956, 31.10.1956, 4.5.1957 et 7.2.1958 (NA), les canaux Ciulinet, 1.12.1956 (NA) et Gradinile, 8.6.1966 (NA).

Bibliographie

Danube : SR 9 : bras Sulina à Sulina (G5). 


\subsubsection{Hydrobiidae Troschel 1857}

\section{Pseudamnicola (Pseudamnicola) penchinati Bourguignat} 1870

Endémique dans la province pontique (seulement dans le Danube inférieur entre Calarasi et l'embouchure).

Eaux à courant lent ; pélophile.

\section{Bibliographie}

Danube : SR 6 et SR 7 : Calarasi-Braila (G3).

Delta du Danube (G3).

\section{Pseudamnicola (Pseudamnicola) razelmiana Grossu 1986}

Endémique dans la province pontique (seulement dans le delta du Danube et le lac Razim).

Eaux stagnantes dulcicoles et faiblement salmastricoles ; pélophile.

\section{Matériel étudié}

Lac Iacob, 16.9.1992 (NA).

\section{Bibliographie}

Delta du Danube : Lacs à Caraorman (G3).

Lacs littoraux de la Mer Noire : Razim (G3).

\section{Pseudamnicola (Pseudamnicola) dobrogica Grossu 1986}

Endémique dans la province pontique (seulement à l'embouchure du Danube dans la Mer Noire).

Eaux à courant lent ; pélophile.

\section{Bibliographie}

Danube : SR 9 : bras Sulina près de l'embouchure (G3).

\section{Pseudamnicola (Pseudamnicola) leontina Grossu 1986}

Endémique dans la province pontique (seulement dans le lac Razim).

Eaux stagnantes faiblement salmastricoles ; pélo- et psammophile.

\section{Bibliographie}

Lacs littoraux de la Mer Noire : Razim (G3).

\subsubsection{Littoridinidae (Gray 1857)}

\section{Potamopyrgus (Potamopyrgus) jenkinsi (Smith 1889)}

Europe (sauf la région nordique). Probablement importée de la Nouvelle Zélande, ou provenant d'une espèce apparentée. Dans le Danube, signalée d'Autriche et Hongrie.

Dans les fleuves, rivières, lacs d'eaux douces ou faiblement salmastricoles ; pélo-, litho- et macrophytophile.

Bibliographie

Lacs littoraux de la Mer Noire : Razim et Sinoie (G3).

\subsubsection{Lithoglyphidae Troeschel 1857}

Lithoglyphus naticoïdes (C. Pfeiffer 1828)
Europe (sauf les régions sud et nord) ; caractéristique dans le bassin du Danube et la province pontique, y compris les bassins du Dniestre, Boug, Dniepr; Donetz et Don.

Dans les fleuves, rivières, ruisseaux, canaux, lacs et bras morts ; litho-, pélo-, psammo- et macrophytophile.

Matériel étudié

Danube.: SR 1 : Moldova Veche, 17.5.1974, 11.7.1974, 22.4.1975 et 2.10.1975 (ZV), Svinita, 7.9.1972 (CS), Mraconia, 28.7.1973, 5.10.1973, 11.4.1974 et 24.4.1975 (ZV), 18.6.1981, 22.9.1981 et 10.8.1982 (PMV), Cerna, 15.5.1973, 23.4.1975 et 30.9.1975 (ZV), 15.8.1989 (NA), Bahna, 27.7.1971 (CS), 11.4.1974 et 1.10.1975 (ZV) et en amont du barrage, 14.10.1971 (CS) ; SR 2 : DrobetaTurnu Severin, 25.3.1972 (CS) ; SR 3 : confluent avec l'Olt, 10.6.1976 (TL) ; SR 6 : Hârsova, 20.6.1962 (PMV) ; SR 7 : bras Macin, 14.4.1962 et 30.6.1962 (PMV) ; SR 8 : confluent avec Siret, 12.6.1976 (TL), Tulcea, 14.8.1976 (TL), Ceatal Izmail, 7.5.1981, 2.6.1981, 12.12.1981 et 15.4.1987 (PMV) ; SR 9 : bras Sulina à Crisan, 18.3.1961 (PMV), à M 2, 20.7.1972 (PMV), à M 1, 11.10.1971, 20.6.1972, 1.5.1974 et 22.7.1976 (PMV) et à $\mathrm{M} \mathrm{O}$, $4.4 .1966,25.9 .1967,8.3 .1968,7.10 .1969,26.8 .1974$ et 20.10.1975 (PMV) et 19.10.1993 (NA) ; SR 10 : Ceatal Sf. Gheorghe, 19.5.1981, 9.8.1981, 20.10.1981, 13.11.1981, 16.4.1982 et 11.6.1982 (PMV), bras Sf. Gheorghe à km 7, 11.7.1966 (PMV), à km 5, 16.4.1967, 5.10.1969, 4.8.1972 et 4.5.1975 (PMV), à km 3, 5.3.1960,

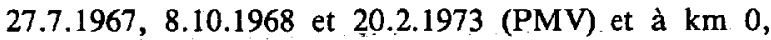
2.5.1971, 8.7.1979 et 4.1.1980 (PMV).

Zone inondable du Danube : ZI 3 : canal Saltava, 10.10.1960, 14.4.1962 (PMV); ZI 4 : canal Filipoiu, 14.4.1962 et 30.6.1962 (PMV) ; ZI 5 : les lacs Crapina, 15.5.1956-7.9.1957, 8.6.1966-13.5.1967, 17.6.1985, 24.9.1989, 11.7 .1990 et 13.9.1992 (NA), Ascunsu, 30.8.1956 et 29.5.1957, Jijila, 18.5.1956-18.4.1958 et 9.9.1966 (NA), Combra, 18.5.1966, 3.10.1956, 4.5.1957 et 7.2.1958 (NA), Popina, 9.6.1966, 8.9.1966, 7.11.1966, 15.5.1967, 17.6.1985 et 13.9.1992 (NA) et Podu, 9.9.1966, 9.11.1966, 12.5.1967 (NA), les canaux Latimile, 1.11.1956, 9.9.1957 (NA), Ciulinet, 1.12.1956 (NA) et Gradinile, 8.6.1966-11.5.1967 (NA).

Delta du Danube : Les lacs Furtuna, 12.5.1967 (ZV) et Iacob, 16.8.1.974 (TL), les canaux Cardon, 28.4.1967 (PMV), Cherhana Sulina, 18.7.1967, 22.7.1976 (PMV), Cherhana Musura, 28.4.1967, 2.10.1967 (PMV), Turetkaia, 17.3.1966, 27.7.1967, 3.10.1979, 9.2.1980 (PMV), et Turcu, 29.9.1967 (PMV), l'île Sacalin, 27.7.1967, 14.10.1974, 6.8.1980 (PMV) et 11.4.1975 (TL).

\section{Bibliographie}

Danube : SR 1 : Pescari, $\mathrm{km} \mathrm{1042,} \mathrm{km} \mathrm{1005;} \mathrm{km}$ 967-968 et Orsova (G5) ; SR 2 : barrage « Portile de Fier II $»(\mathrm{~N})$; confluent avec Jiu (N) ; SR 3 : confluent avec l'Olt (N) ; SR 4 : Giurgiu (G5) ; SR 6 : Jirlau-Borcea 
Dervent (G5), Hârsova (N) ; SR 7 : bras Macin (N) ; SR 8: Ceatal Izmail (P1) ; SR 9 : bras Tulcea (P1), bras Sulina à Maliuc, $M$ 22, M 19, M 14, M 9, M 3, M 2, et à M 0 (G5) ; SR 10 : Ceatal Sf. Gheorghe (G5), bräs Sf. Gheorghe à Mahmudia, Uźlina, km 5 et km 0 (GS); ; canal . Turcu (N) ; bras Chilia (MK).

Zone inondable du Danube : ZI 2 lac Oltina (P.3); ZI 3 : canal Saltava et lacs (P5) ; ZI 4 : canal Filipoiu (P5).

Delta du Danube : Lac Gorgova (G5).

\section{Lithoglyphus apertus (Küster 1852)}

Espèce pontique-danubienne endémique (Danube moyen et inférieur, Drava et Sava).

Eaux à courant lent (fleuves, rivières) ; litho-, psammoet pélophile.

Bibliographie

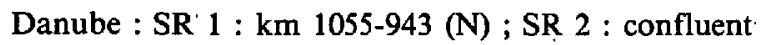
avec Jiu (N) ; SR 4 : Giurgiu (G3) ; SR 5 : Calarasi (G3).

Zone inondable du Danube : ZI 2 : Lac Oltina (P3).

\section{Lithoglyphus pygmaeus Frauenfeld 1863}

Espèce pontique-danubienne endémique (Danube moyen et inférieur, Drava et Sava).

Eaux à courant lent (fleuves, rivières); litho- et pélophile.

Bibliographie

Gastéropode : SR 4 : Giurgiu (coquilles dans les alluvions et individus vivants dans les sondages hyporhéiques!) (G3).

\subsubsection{Bithyniidae Gray 1849}

\section{Bithynia (Bithynia) tentaculata (Linnaeus 1758)}

Paléarctique (euro-sibérienne avec centre de l'aire de répartition en Europe). Tout le bassin du Danube.

Fleuves; rivières et lacs; litho-, pélo- et macrophytophile.

Matériel étudié

Danube : SR 1 : Moldova Veche, 2.10.1975 (ZV) et Mraconia, 7.5.1981, 28.10.1981, 18.8.1982 (PMV) ; SR 9 ; bras Sulina à Sulina, 11.6.1967 (ZV), 16.10.1993 (NA) ; SR 10 : bras Sf. Gheorghe, 8.8.1979, 12.12.1981, 14.5.1982 (PMV).

Zone inondable du Danube : ZI 5 : les lacs Crapina, 15.5.1956-7.9.1957, 8.6.1966-13.5.1967 (NA), Ascunsu 30.8.1956, 29.5.1967 (NA), Jijila, 18.5.1956-18.4.1958 (NA), Combra, 18.5.1956, 3.10.1956, 4.5.1957, 7.2.1958 (NA), les canaux Latimile, 1.11.1956, 9.9.1957 (NA), Ciulinet, 1.12.1956 (NA) et Gradinile, 8.6.1966-11.5.1967.

Delta du Danube : Les lacs Furtuna, 12.5.1957, 16.9.1967 (ZV), Trei Iezere, 8.6.1972, 4.11.1972 (PM), Matita, 29.10.1971 (PM), Merhei, 12.10.1967 (PMV) et lacob, 5.6.1974, 18.10.1974 (TL), les canaux Imputita,
20.6.1963, 24.12.1963, 13.7.1964, 16.2.1965, 11.5.1965, 18.9.1965, 17.5.1968 (PMV), Gafon, 13.5.1967 (ZV), Porcu, 29.8.1962, 20.6.1963, 30.9.1963, 22.12.1963, 2.4.1965, 20.6.1965, 18.9.1965, 21.12.1965 (PMV) et Turetkaia, 27.7.1967 (PMV), zone Maliuc, 14.8.1967 (ZV), zone Carasuhat, 10.6.1967, 17.9:1967 (ZV), île Sacalin, 28.7.1979, 9.4.1980, 8.10.1980 (PMV).

Bibliographiè

Danube : SR 1 ; Cerna et Bahna (N) ; SR 2 : barrage " Portile de Fier. II » (NA) ; SR 4 : Giurgiu (G5); SR 6 : Jirlau-Borcea et Dervent (G5) ; SR 8 : Ceatal Izmail (N) ; SR 9 : bras Tulcea (G5), bras Sulina à Maliuc; Gorgova, M 14, M 9, M 2, M 0 (G5) et à Crisan (F) ; SR 10 : Ceatal Sf. Gheorghe (G5), bras Sf. Gheorghe à Perivolquvca (G5).

Zone inondable du Danube : ZI 1 : les petits lacs du km 1032, km 1015 (Cozla) et km 961 (Ieselnita) (N) ; ZI 2 : lac Oltina (P3) ; ZI 3 : île Borcea (P5) ; ZI 4 : canal Filipoiu (P5).

Delta du Danube : Les lacs Gorgova, Matita, Merhei et Rosu (G7), Stambulet et Rosulet (N). Egalement bras et delta Chilia (MK).

\section{Bithynia (Bithynia) leachi (Scheppard 1823)}

Paléarctique (euro-sibérienne, jusqu'au Kamtchatka). Dans le Danube, de la Slovaquie jusqu'à l'embouchure dans la Mer Noire.

Eaux stagnantes et à courant lent et aussi salmastricoles ; pélo- et macrophytophile.

Matériel étudié

Danube : SR 9 : Sulina, 20.9.1992 (NA).

Bibliographie

Danube : SR 9 : bras Sulina à M 22 (Bratul Vechi) et à M 2 (G5).

Zone inondable du Danube : ZI 3 : lacs du complexe Borcea de Jos $(\mathrm{N})$.

Delta du Danube : Canal Imputita (G3), lac Raducu (N).

Lacs littoraux de la Mer Noire : Golovita (G3).

\subsubsection{Micromelaniidae Thiele 1925}

\section{Micromelania (Turricaspia) lincta Milaschewitch 1908}

Endémique dans la province pontique (Delta du Danube, lac Razim, estuaires des Dniester et Boug).

Eaux stagnantes dulcicoles et faiblement salmastricoles ; psammo- et pélophile.

Bibliographie

Danube : SR 9 : embouchure dans la Mer Noire (G3). Delta du Danube (G3).

Lacs littoraux de la Mer Noire : complexe des lacs Razim (G3). 
Micromelania (Turricaspia) ostroumovi Golikov \& Starobogatov 1966

Endémique dans la province pontique. Seulement dans le delta du Danube.

Eaux stagnantes dulcicoles et faiblement salmastricoles ; pélophile.

\section{Bibliographie}

Delta du Danube : Lacs (matériel vivant) et forages géologiques (état subfossile) (G3).

Micromelania (Turricaspia) ismailensis (Golicov \& Starobogatov 1966)

Endémique dans la province pontique (seulement à l'embouchure du Danube) et dans les lacs du sud de la Bessarabie : Ialpoug et Katalpoug).

Eaux stagnantes ou à courant lent, dulcicoles ou faiblement salmastricoles ; pélophile.

Bibliographie

Danube : SR 9 : Sulina (G3).

\subsubsection{Thiaridae Troschel 1857}

\section{Esperiana (Microcolpia) acicularis (Férussac 1829)}

Syn. Fagotia (M.) acicularis (Fér. 1823) sensu Frank et al. 1990.

Pontique-danubienne (Dniepr, Boug, Dniester et le bassin du Danube de l'Autriche jusqu'à l'embouchure).

Eaux courantes (fleuves, rivières, ruisseaux) et stagnantes (lacs) ; pélo- et macrophytophile, mais aussi lithophile.

Matériel étudié

Danube : SR 9 : bras Sulina à l'embouchure, 4.4.1966 (PMV) ; SR 10 : bras Sf. Gheorghe à km 1, 6.3.1968, 4.1.1980 ((PMV).

Zone inondable du Danube : ZI 3 : canal Saltava, 10.10.1960 (PMV) ; ZI 4 : canal Filipoiu, 16.4.1962, 30.6.1972 (PMV); ZI 5: les lacs Crapina, 15.5.1956-7.9.1957, 8.6.1966-13.5.1967, 17.6.1985, 11.7.1990 (NA), Ascunsu, 30.8.1956, 21.5.1957 (NA), Jijila, 18.5.1956-18.4.1958, 9.9.1966 (NA), Podu, 9.9.1966, 12.5.1967 (NA), les canaux Latimile, 1.11.1956, 9.9.1957 (NA), Ciulinet, 1.12.1956 (NA) et Gradinile, 1.5.1957, 17.6.1985 (NA).

Delta du Danube : Canal Turetkaia, 6.3.1968 (PMV).

Bibliographie

Danube : SR 1 : Moldova Veche (N) ; SR 6 : JirlauBorcea et Dervent (G5) et Hârsova (N) ; SR 7 : bras Macin (N) ; SR 9 : bras Sulina à Maliuc, M 9, M 2 et port Sulina (G2 et G5) ; SR 10 : bras Sf. Gheorghe (G2).

Delta du Danube : Canal Turcu (N); bras et delta de Chilia (MK).

Esperiana (Fagotia) esperi (Férussac 1829)

Syn. Fagotia (M.) esperi (Fér. 1823) sensu Frank et al. 1990
Pontique-danubienne (Dniepr, Boug, Dniester et le bassin du Danube de l'Autriche jusqu'à l'embouchure).

Eaux courantes (fleuves, rivières, rujsseaux) et stagnantes (lacs) ; associée à $E$. acicularis ; pélo- et macrophytophile, mais aussi lithophile.

Matériel étudié

Danube : SR 9 : bras Sulina à Sulina, 20.10.1975 (PMV) et 21.9.1992 (NA) ; SR 10 : bras Sf. Gheorghe à $\mathrm{km} \mathrm{7}$, 11.7.1966 (PMV), à km 5, 5.10.1969, 4.8.1972, 29.5.1974 (PMV), à km 3, 5.3.1968, 20.5.1974 (PMV), à km 1, 6.3.1968, 8.7.1979, 4.1.1980 (PMV).

Zone inondable du Danube : ZI 3 : canal Saltava, 10.10.1960 (PMV); ZI 5: les lacs Crapina, 15.5.1956-7.9.1957, 8.6.1966-13.5.1967, 17.6.1985, 24.9.1989, 11.7.1990, 13.9.1992 (NA), Ascunsu, 30.8.1956, 29.5.1957 (NA), Popina, 9.6.1966, 8.9.1966, $7.11 .1966, \quad 15.5 .1967, \quad 11.7 .1990$ (NA), Jijila, 18.5.1956-18.4.1958 (NA), Combra, 18.5.1956, 3.10.1956, 4.5.1957, 7.2.1958 (NA) et Podu, 9.9.1966, 9.11.1966, 12.5.1967 (NA), les canaux Latimile, 1.11.1956, 9.9.1957 (NA), Ciulinet, 1.12.1956 (NA) et Gradinile, 8.6.1966-11.5.1967 (NA).

Delta du Danube : Les canaux Cherhana Musura, 2.10.1967 (PMV), Turetkaia, 27.7.1967, 6.3.1968, 3.10.1979, 6.8.1980 (PMV) et Turcu, 9.4.1980 (PMV), île Sacalin, 4.4.1966, 27.8.1967, 7.10.1968, 4.4.1970, 29.8.1974, 14.10.1974, 4.6.1975, 1.8.1977, 3.10.1979, 9.4.1980, 5.10.1980 (PMV), 18.7.1976 (TL), 18.5.1977, 18.7.1977, 14.9.1977 (AE).

\section{Bibliographie}

Danube : SR 4 : Giurgiu (G5) ; SR 6 : Jirlau-Borcea (G5) ; SR 9 : bras Sulina à Maliuc, M 24, Gorgova, $M$ 19, M 9, M 2 et à l'embouchure (G5); S 10 : Ceatal Sf. Gheorghe et bras Sf. Gheorghe à km 5 (G5).

Zone inondable du Danube : ZI 3 : canal Saltava (P5).

Delta du Danube : Les lacs Gorgova et Rosu (P2), canal Cherhana Sulina et le golfe Musura (P2). Egalement dans le bras et le delta de Chilia (MK).

\subsection{Basommatophora A. Schmidt 1855}

\subsubsection{Physidae Fitzinger 1833}

\section{Physa fontinalis (Linnaeus 1758)}

Holarctique avec centre de l'aire de répartition en Europe (sauf région nordique). Tout le bassin du Danube.

Eaux stagnantes (lacs, bras morts) et courantes (fleuves, rivières) riches en végétation ; psammo- pélo- et macrophytophile.

Matériel étudié

Danube : SR 9 : bras Sulina à Sulina, 17.9.1992 (NA).

Delta du Danube : Lac Furtuna, 8.6.1967 (ZV), canal Cherhana Musurā, 2.10.1967 (PMV), zone Carasuhat, 
10.6.1967 (ZV), golfe Musura, 3.10.1967 (PMV), île Sacalin, 18.7.1976 (TL), 18.7.1977, 14.9.1977 (AE).

Bibliographie

Danube : SR 1 : défilế du Danube à km 1054 et 1031 (N) ; SR 9 : bras Sulina à Crisan (F).

Zone inondable du Danube : ZI 1 : petit lac Cozla (N) ; ZI 2 : lac Oltina $(\mathrm{N})$.

Delta du Danube : Zone Maliuc (N).

Physa acuta (Draparnaud 1805)

Circumméditerranéenne, ouest et est européenne. Danube et son bassin de l'Autriche à l'embouchure.

Lacs et rivières à courant lent riches en végétation ; pélopsammo- et macrophytophile.

Matériel étudié

Danube : SR 1 : Mraconia, 9.3.1972, 15.10.1974 (CS) et 7.6.1972 (PMV) ; SR 10 : bras Sulina à M O, 15.7.1975 (TL) et 21.9.1992 (NA).

Delta du Danube : Canal Cherhana Musura, 2.10.1967 (PMV) ; canal Turetkaia, 4.6.1980 (PMV) ; île Sacalin, 18.10.1975, 1.11.1975, 5.8.1980, 5.10.1980 (PMV).

Bibliographie

Danube : SR 1 : lac de barrage « Portile de Fier I » à km 1054, km 1025, km 1018 et à Mraconia (N) ; SR 2 : confluent avec Jiu (G5) ; SR 5 : Calarasi (G4); SR 6 : Dervent (G5) ; SR 9 : bras Sulina à M 3 (G5) ; SR 10 : Ceatal Sf. Gheorghe (N), bras Sf. Gheorghe à M $3(\mathrm{~N})$.

Zone inondable du Danube : ZI 2 : lac Oltina (G4) ; ZI 3 : île Borcea $(\mathrm{N})$.

\subsubsection{Lymnaeidae Rafinesque 1815}

\section{Lymnea stagnalis (Linnaeus 1758)}

Holarctique. Tout le bassin du Danube.

Lacs, bras morts et rivières à courant lent, riches en végétation submergée ; macrophytophile et hyponeustonique.

\section{Matériel étudié}

Danube : SR $9:$ bras Sulina à M 1, 16.9.1992 (NA). Zone inondable du Danube : ZI 5 : les lacs Crapina, 28.6.1957 (NA), Jijila, 4.7.1956, 4.3.1957 (NA), Combra, 4.5.1957 (NA) et Somovelu, 2.2.1958 (NA).

Delta du Danube : Canal Cherhana Musura, 25.9.1967 (PMV) ; bras et delta Chilia (MK).

Bibliographie

Danube : SR 6 : Jirlau-Borcea (G5) ; SR 9 : bras Sulina à Crisan (F), à M 9 et M. 3 (G5); SR 10 : bras Sf. Gheorghe à km 53 (G5).

Zone inondable du Danube : ZI 2 : lac Oltina $(N) ; Z 1$ 3 : île Borcea (N).

Stagnicola (Stagnicola) palustris (O.F. Müller 1774)

Holarctique. Tout le bassin du Danube.
Lacs grands et petits, bras morts et rivières à courant lent, riches en végétation, aquatique ; macrophytophile. Matériel étudié

Danube : SR 9 : bras Sulina à Maliuc, 13.5.1967 (ZV) et à Sulina, 14.9.1992 (NA).

Delta du Danube : Canal Imputita, 20.5.1963 (PMV).

Bibliographie

Danube : SR 9 : bras Sulina à Crisan (F) et à M 2 (G5).

Zone inondable du Danube : ZI 1 : petits lacs à $\mathrm{km} 1032$ et à $\mathrm{km} 1000(\mathrm{~N})$ et petit lac Ieselnita $(\mathrm{N})$; ZI 3 : île Borcea $(\mathrm{N})$.

Delta du Danube : Lac Porcu et lacs dans la zone Maliuc (N).

\section{Stagnicola (Stagnicola) corvus (Gmelin 1791)}

Paléarctique. Tout le bassin du Danube.

Lacs, bras morts, rivières et fleuves à courant lent, riches en végétation (conditions optimales dans le Danube et son système de lacs et canaux) ; macrophytophile.

Bibliographie

Danube : SR 9 : bras Sulina à Crisan (F).

Delta du Danube : lacs de la zone Caraorman (F).

Lacs littoraux de la Mer Noire (complexe Razim) : Babadag (G4).

Stagnicola (Stagnicola) turricula (Held 1836) sensu Jackiewicz 1959

Paléarctique. Tout le bassin du Danube.

Lacs (grands et petits), bras morts, canaux, rivières et fleuves riches en végétation ; macrophytophile.

Bibliographie

Danube : SR 9 : bassin Sulina à Crisan $(\mathrm{F})$.

Radix (Radix) auricularia (Linnaeus 1758).

Paléarctique. Tout le bassin du Danube.

Lacs, bras morts, fleuves et rivières à courant lent, riches en végétation; pélo- et macrophytophile.

Bibliographie

Zone inondable du Danube : ZI 5 : lac Crapina (G4). Delta du Danube : « nombreux lacs » (G4).

Radix (Radix) ovata (Draparnaud 1805)

Paléarctique. Tout le bassin du Danube.

Lacs grands et petits, bras morts, fleuves et rivières à courant- lent, riches en végétation; pélo- et macrophytophile.

Matériel étudié

Danube : SR 9 : bras Sulina à M O, 15.7.1975 (TL) ; SR 10 : bras Sf. Gheorghe à km O, 5.8.1980 (PMV).

Zone inondable du Danube : ZI 5 : les lacs Crapina, 29.9.1956, 3.5.1957, 8.9.1966, 7.11.1966, 11.7.1990 (NA), Jijila, 3.6.1957, 8.4.1958 ((NA) et Popina, 8.9.1966 (NA). 
Delta du Danube : les canaux Cardon 18.5.1968 (PMV) et Imputita, 17.5.1968 (PMV), île Sacalin, 18.7.1977 (AE), 3.10.1979 et 6.8.1980 (PMV).

\section{Bibliographie}

Danube : SR 9 : bras Sulina à Crisan (F), M 3, M 2 et à l'embouchure (G5).

Zone inondable du Danube : ZI 1 : petits lacs à $\mathrm{km}$ $1000, \mathrm{~km}$ 976, Mraconia et Ieselnita (N) ; ZI $2:$ lac Oltina (P3) ; ZI 3 : île Borcea (P5) ; ZI 5 : lac Crapina (G4).

Delta du Danube : lac Puiu et Rosulet (N).

\section{Radix (Radix) peregra (O.F. Müller 1774)}

Paléarctique. Tout le bassin du Danube.

Lacs, étangs, bras morts, rivières à courant lent ; caractéristique des zones marécageuses; pélo- et macrophytophile.

\section{Matériel étudié}

Danube : SR 9 : bras Sulina à M O, 4.6.1975 (TL).

Delta du Danube : les canaux Cardon, 28.4.1967 (PMV), 22.7.1976 (TL) et Musura, 22.7.1976 (TL), golfe Musura, 22.7.1976 (TL), île Sacalin, 10.8.1968, 4.4.1970, 18.10.1975, 9.9.1976 (PMV), 18.8.1977 et 14.9.1977 (AE).

\section{Bibliographie}

Danube : SR 1 : défilé du Danube à $\mathrm{km} \mathrm{1034,} \mathrm{km} 1031$, $\mathrm{km} \mathrm{1023,} \mathrm{km} 1018$ et à Mraconia $(\mathrm{N})$.

Zone inondable du Danube : ZI 1 : petits lacs à Cozla et Dubova (N) ; ZI 2 : lac Oltina (P3) ; ZI 3 : île Borcea (P5).

Delta du Danube : les lacs Gorgova, Uzlina, Rosu (G7) et Zatonul Mic (N) et le canal Imputita (G7).

\section{Galba (Galba) truncatula (O.F. Müller 1774)}

Holarctique. Tout le bassin du Danube.

Caractéristique de petits milieux temporaires, mais aussi dans les lacs, étangs, sources etc...; pélo- et macrophytophile.

\section{Bibliographie}

Danube : SR 9 : bras Sulina à M 2 (G5).

\subsubsection{Ancylidae Rafinesque 1815}

\section{Ancylus fluviatilis O.F. Müller 1774}

Paléarctique avec centre de l'aire de répartition en Europe. Tout le bassin du Danube.

Caractéristique des eaux courantes (fleuves et rivières à lit pierreux avec couverture biologique, mais aussi dans les eaux stagnantes avec végétation aquatique; lithorhéophile et pélo-macrophytophile.

Matériel étudié

Danube : SR 1 : confluent avec Mraconia, 9.7.1974, 1.8.1975 (ZV), 28.7.1989 (NA).
Delta du Danube : lac Iocob, 5.6.1974 (TL), canal Marcu, 21.7.1976 (TL), 7.9.1976 (PMV), golfe Musura, 7.9.1976 (PMV) et île Sacalin, 14.9.1.977 (AE).

Bibliographie

Danube : SR 1 : confluent avec les rivières Plavisevita ( $k m$ 976), Mraconia (km 967), Ieselnita (km 961), Cerna $(\mathrm{km} \mathrm{954)}$ et Vodita $(\mathrm{km} \mathrm{953)}(\mathrm{N})$.

Zone inondable du Danube : ZI 1 : les petits lacs Camenita $(\mathrm{km} \mathrm{1025),} \mathrm{Cozla}(\mathrm{km} \mathrm{1015)}$ et Ieselnita $(\mathrm{km} \mathrm{961)} \mathrm{(N).}$

Delta du Danube : les lacs Zatonul Mic, Raducu et Puiu (N).

\subsubsection{Ferrissidae Zilch 1960}

\section{Ferrissia wautieri (Mirolli 1960)}

Ouest et centro-européenne, y compris le nord de la péninsule balcanique. Danube : Autriche, Hongrie et Roumanie seulement.

Eaux stagnantes (lacs, étangs, marais) et courantes, riches en végétation; lithophile et macrophytophile.

Matériel étudié

Danube : SR 9 : bras Sulina à M O, 18.9.1992 (NA). Bibliographie

Danube : SR 9 : bras Sulina à Sulina et Dunarea Veche (G6).

Zone inondable du Danube : ZI 4 : lac Corotisca (G4).

\subsubsection{Acroloxidae Thiele 1931}

Acroloxus lacustris (Linnaeus 1758)

Paléarctique (euro-sibérienne). Tout le bassin du Danube.

Lacs, bras morts, étangs, fleuves riches en végétation, macrophytophile (surtout), litho- et pélophile.

Matériel étudié

Delta du Danube : lac Porcu, 19.3.1961, 29.8.1962, 31.7.1963, 30.9.1963, 22.7.1965, 21.12.1965 (PMV) et 13.9.1992 (NA), canal Imputita, 31.7.1963, 10.8.1964, 11.5.1965, 20.8.1965, 21.10.1965, 21.12.1965 et 21.7.1967 (PMV) et 13.9.1992 (NA), canal Busurca, 19.3.1961 (PMV), île Sacalin, 3.10.1979 et 8.10.1980 (PMV).

Bibliographie

Danube : SR 9 : bras Sulina à M 22, M 3, M 2 et à l'embouchure (G5) et à Crisan (F).

Zone inondable du Danube : ZI 2 : lac Oltina (P3) ; ZI 3 : île Borcea (P5).

Delta du Danube : lac Zatonu Mic (N).

\subsubsection{Planorbidae Rafinesque 1815}

Planorbis planorbis (Linnaeus 1758)

Holarctique avec centre de l'aire de répartition en Europe et ouest de l'Asie. Tout le bassin du Danube. 
Eaux stagnantes (lacs, étangs, bras morts, petits milieux temporaires et eaux à courant lent, riches en végétation ; pélo- et macrophytophile.

Matériel étudié

Danube : SR 9 : bras Sulina à Maliuc, 11.6.1967 (ZV), à M 0, 4.6.1975 (PMV) et à Sulina, 18.9.1992 (NA) ; SR $10:$ bras Sf. Gheorghe à $\mathrm{km} 0,4.1 .1980$ et 3.6.1980 (PMV).

Delta du Danube : canal Imputita, 8.10.1968 (PMV), canal Turetkaia, 4.6.1960 (PMV), golfe Musura, 22.7.1976 (TL), île Sacalin, 28.7.1979 et 9.4.1980 (PMV).

Bibliographie

Danube : SR 1 : lac du barrage «Portile de Fier I" (N) ; SR $4:$ Giurgiu (N) ; SR 6: Jirlau-Borcea et Dervent (G5) ; SR 9 : bras Sulina à Gorgova, M 19, M 3, M 2, à l'embouchure (G5) et à Crisan (F).

Zone inondable du Danube : ZI 1 : petits lacs à $\mathrm{km}$ 1032, km 1025 (Camenita), km 1015 (Cozla), km 1000 et km 961 (Ieselnita) (N) ; ZI 2 : lac Oltina (P3) ; ZI 3 : île Borcea (N).

Delta du Danube : les lacs Matita et Merhei (N), les lacs deś zones Maliuc (N) et Caraorman ( $\mathrm{F}$ ), le canal Turcu (N).

\section{Planorbis carinatus O.F. Müller 1774}

Paléarctique (euro-sibérienne, jusqu'à l'Amour). Tout le bassin du Danube.

Caractéristiques des eaux stagnantes (lacs, bras morts) et à courant lent (canaux, nivières, fleuves), riches en végétation ; pélo- et macrophytophile.

Matériel étudié

Danube : SR 9 : bras Sulina à M 1, 22.9.1992 (NA).

Bibliographie

Danube : SR 9 : bras. Sulina à Maliuc, $M$ 24, $M 9$ et M 2 (G5) et à Crisan (F).

Delta du Danube (G4).

Anisus (Anisus) septemgyratus (Rossmässler 1835)

Europe (sauf régions situées à l'extrême nord et sud) avec centre de l'aire de répartition à l'est. Danube et ses affluents, de l'Autriche jusqu'à l'embouchure.

Petits milieux stagnants (lacs, marais) à végétation ; macrophytophile.

Matériel étudié

Delta du Danube : canal Imputita, 21.12.1963, 21.7.1965 et 18.9.1965 (PMV), golfe Musura, 21.7.1965 (PMV).

\section{Bibliographie}

Danube : SR 9 ; bras Sulina à Crisan (F).

Anisus (Anisus) spirorbis (Linnaeus 1758)
Paléarctique avec centre de l'aire de répartition en Europe et ouest de l'Asie. Tout le bassin du Danube.

Lacs petits ou moyens, permanents ou temporaires et bras morts avec végétation; pélo- et macrophytophile.

Matériel étudié

Danube : SR 9 : bras Sulina à Sulina, 21 9.1992 (NA).

Delta du Danube : lac Puiu, 8.6.1967, 3.5.1968 et 12.9.1969 (SC), lac Stambulet, 9.6.1967, 5.5.1968 et 10.9.1969 (SC).

Bibliographie

Danube : SR 1 : Berzasca (N) ; SR 4": Giurgiu (G5); SR 9 : bras Sulina à M 3, M 2 et à l'embouchure (G5).

Zone inondable du Danube : ZI 2 : lac Oltina (P3); ZI 3 : île Borcea (N).

Delta du Danube (G4).

Anisus (Anisus) rotundatus (Poiret 1801)

Syn. A. leucostomus (Mil. 1813) sensu Frank et al. 1990

Paléarctique avec centre de l'aire de répartition en Europe et ouest de l'Asie. Tout le bassin du Danube.

Petits lacs permanents ou temporaires, marais, bras morts, canaux, fleuves et rivières à courent lent, riches en végétation; pélo- et macrophytophile.

Matériel étudié

Delta du Danube : canal Imputita, 13.9.1992 (NA).

Bibliographie

Danube : SR 9 : bras Sulina à Crisan (F).

Anisus (Disculifer) vortex (Linnaeus 1758)

Ouest- et centro paléarctique (Europe et ouest de l'Asie).

Tout le bassin du Danube.

Eaux temporaires, bras morts, étangs, marais, canaux, lacs etc, riches en végétation; macrophytophile.

Matériel étudié

Delta du Danube : lac Iacob, 5.6.1974 (TL), canal Imputita, 18.9.1965 (PMV) et 13.9.1992 (NA).

Bibliographie

Danube : SR 9 : bras Sulina à Crisan (F).

Zone inondable du Danube : ZI 5 : lac Crapina (G4).

Anisus (Disculifer) vorticulus (Troschel 1834)

Ouest- centro- et est européenne. Tout le Danube et ses systèmes lacustres.

Eaux stagnantes et à courant lent, riches en végétation ; macrophytophile.

Bibliographie

Danube : SR 9 : bras Sulina à Crisan (F).

Zone inondable du Danube: ZI 3 : île Borcea (N).

Delta du Danube (G4). 
Bathyomphalus contortus (Linnaeus 1758)

Paléarctique. Tout le bassin du Danube.

Eaux stagnantes et à courant lent, riches en végétation ; macrophytophile.

Bibliographie

Delta du Danube : «quelques exemplaires trouvés dans le delta du Danube » (G4).

\section{Gyraulus (Gyraulus) albus (O.F. Müller 1774).}

Holarctique. Tout le bassin du Danube.

Eaux stagnantes permanentes et eaux à courant lent ; conditions optimales dans les bras morts riches en végétation; dans les eaux roumaines du Danube : lithopsammo- pélo- et macrophytophile.

Matériel étudié

Zone inondable du Danube : ZI 5: lac Crapina, 15.5.1956-31.10.1956, 25.1.1957-11.11.1957, 17.6.1985 et 11.7.1990 (NA), lac Jijila, 18.5.1956-4.11.1956, 27.1.1957-7.11.1957 et 7.2.1958 (NA), lac Combra, 18.5.1956, 3.10.1956, 4.5.1957 et 7.2.1958 (NA), canal Latimile, 1.11 .1956 et 9.9.1957 (NA).

Delta du Danube : les lacs Raducu, Puiu, Rosulet et Stambulet, 8.5.1967-10.9.1969 (SC).

Lacs littoraux de la Mer Noire (complexe Razim): Istria, 10.10.1961 (PMV).

\section{Bibliographie}

Danube : SR 1 : Ieselnita et Bahna (N) ; SR 4 : Giurgiu (G5) ; SR 6 : Jirlau-Borcea, Dervent et Harsova (G5) ; SR 9 : bras Sulina à Gorgova, M 19, M 2, à l'embouchure (G5) et à Crisan (F) ; SR 10 : Ceatal Sf. Gheorghe et bras Sf. Gheorghe à $\mathrm{km} 53$ (G5 et P1).

Zone inondable du Danube : ZI 1 : les petits lacs Cozla, Dubova et Ieselnita (N) ; ZI 2 : lac Oltina (P3) ; ZI 3 : île Borcea (P5).

Delta du Danube : zone Pardina (P4), les lacs Matita, Merhei, Rosu, Uzlina et Gorgova (G7 et P2).

\section{Gyraulus (Gyraulus) acronicus (Férussac 1807)}

Holarctique. Le Danube et ses affluents : Allemagne, Autriche et Roumanie seulement.

Eaux stagnantes et à courant lent, près des rives ; macrophytophile.

\section{Bibliographie}

Danube : SR 9 : bras Sulina à Crisan (F).

\section{Gyraulus (Torquis) laevis (Alder 1838)}

Holarctique. Tout le bassin du Danube.

Eaux stagnantes et à courant lent, riches en végétation ; pélo- psammo- et macrophytophile.

\section{Bibliographie}

Danube : SR 1.: lac du barrage "Portile de Fier I " entre $\mathrm{km} 1055$ et $943(\mathrm{~N})$.
Zone inondable du Danube : ZI 2 : lac. Oltina (P3). Delta du Danube (G4).

Armiger crista (Linnaeus 1758)

Syn. Gyraulus crista (L. 1758) sensu Frank et al. 1990

Holarctique : avec centre de l'aire de répartition en Europe et ouest de l'Asie. Tout le bassin du Danube.

Eaux stagnantes permanentes, temporaires et eaux à courant lent, riches en végétation ; macrophytophile.

Matériel étudié

Delta du Danube : les lacs Iacob, 16.8.1974 (TL), Porcu, 20.6.1963, 30.9.1963, 22.12.1963, 2.4.1965 et 18.9.1965 (PMV), Rosu et Rosulet, 8.5.1967-10.9.1969 (SC) et Zatonul Mic, 3.5.1976 (SC), canal Imputita, 11.5.1965, 20.8.1965, 21.10.1965 et 21.12.1965 (PMV).

\section{Bibliographie}

Danube : SR 9 : bras Sulina à Gorgova, M 19, M 12, M 2 (G5) et à Crisan (forma cristatus, F).

Delta du Danube : lac Gorgova (G4).

\section{Segmentina nitida (O.F. Müller 1774)}

Ouest paléarctique. Tout le bassin du Danube.

Eaux stagnantes peu profondes permanentes ou temporaires et eaux à courant lent, riches en végétation; macrophytophile.

Matériel étudié

Danube : SR 10 : bras Sf. Gheorghe à km 0, 28.10.1971 (PMV).

Delta du Danube : lac Iacob, 16.8.1974 et 18.10.1974 (TL), lac Porcu, 18.9.1965, 23.11.1965 (PMV) et 17.9.1992 (NA), canal vers le lac Porcu, 29.8.1962 (PMV), canal Imputita, 31.3.1965 et 16.6.1965 (PMV).

Bibliographie

Delta du Danube : canal Imputita (G4).

\section{Hippeutis complanatus (Linnaeus 1758)}

Ouest et centro paléarctique (Europe, ouest de l'Asie jusqu'au Ienisseï et nord de l'Afrique). Tout le bassin du Danube.

Eaux stagnantes permanentes ou temporaires et eaux à courant lent, riches en végétation ; macrophytophile.

Matériel étudié

Danube : SR 9 : bras Sulina à Sulina, 14.9.1992 (NA).

Delta du Danube : lac Iacob, 16.8.1974 (TL).

Bibliographie

Danube : SR 9 : bras Sulina à M 9, M 3 (G5) et à Sulina (G4), bras Dunarea Veche (G4).

Delta du Danube : lacs sur le cordon Caraorman (G4). et canal Imputita (G4).

Lacs littoraux de la Mer Noire (complexe Razim): Babadag et Golovita à Jurilovca (G4). 
Planorbarius corneus (Linnaeus 1758)

Ouest et centro paléarctique (Europe, Anatolie et Asie jusqu'aux monts Altai). Tout le bassin du Danube.

Eaux stagnantes permanentes ou temporaires et eaux à courant lent ; conditions optimales dans les bras morts riches en végétation; pélo- et macrophytophile.

Matériel étudié

Danube : SR 10 : bras Sf. Gheorghe à $\mathrm{km} 0,4.6 .1980$ et 5.8.1980 (PMV).

Zone inondable du Danube: ZI 3 : canal Saltava, 10.10.1960 (PMV); ZI 5: lac Crapina, 15.5.1956, 30.4.1957, 31.5.1957, 30.7.1957, 17.6.1985 et 11.7.1990 (NA), lac Jijila, 4.11.1956, 27.1.1957, 4.5.1957 et 3.10.1957 (NA), lac Combra, 3.10.1956 (NA).

Delta du Danube : lac Trei Iezere, 4.10.1972 (PMV), canal Imputita, 8.10.1968 et 7.8.1968 (PMV), île Sacalin, 28.7.1979, 3.10.1979, 9.4.1980 et 8.10.1980 (PMV).

\section{Bibliographie}

Danube : SR 4 : Giurgiu (G4) ; SR 6 : Dervent (G5) ; SR 9 : bras Sulina à M 9, M 3, M 2 (G5) et à Crisan (F).

Zone inondable du Danube : ZI 1 : petits lacs à km 1032, km 1025 (Camenita), km 1015 (Cozla), km 1000 et km 961 (Ieselnita) (N) ; ZI 2 : lac Oltina (P3) ; ZI 3 : île Borcea (P5).

Delta du Danube : zone Pardina (P4), les lacs Rosu, Uzlina et Gorgova (G7). Bras et delta Chilia (MK).

\section{Remarques zoogéographiques et paléo- biogéographiques}

On connaît, jusqu'ici, dans la faune du secteur roumain du Danube (y compris la zone inondable et le delta), 54 espèces de Gastéropodes qui appartiennent à 3 ordres : Neritoidea (5 espèces), Mesogastropoda (21 espèces) et Basommatophora (28 espèces). On peut distinguer 13 catégories zoogéographiques.

\subsection{Espèces endémiques dans la province pontique}

Jusqu'à présent, il a été décrit 8 espèces appartenant à 3 genres qui ont dans cette province une aire de répartition restreinte ou très restreinte : Theodoxus euxinus (embouchure du Danube et lac Razim), Pseudamnicola penchinati (Danube depuis Calarasi jusqu'à Sulina), $P$. razelmiana (Danube et lac Razim), $P$. dobrogica (embouchures du Danube), $P$. leontina (lac Razim), Micromelania lincta (delta du Danube, lac Razim, estuaires du Dniester et du Boug). M. ostroumovi (lacs du delta du Danube), $M$. ismailensis (embouchures du Danube et lacs Ialpoug et Katalpoug).

Il s'agit là d'espèces relictes qui avaient autrefois dans le bassin pontique une aire de répartition plus large. $T$. euxinus vivait pendant le pliocène dans toutes les eaux salmastricoles de ce bassin. $M$. ostroumovi a été trouvé dans la même station du delta du Danube sous forme d'individus vivants et de coquilles subfossiles.

\subsection{Espèces endémiques pontiques-caspiennes}

Une seule espèce : Theodoxus pallasi, trouvée à l'embouchure du Danube. C'est un néritide connu des eaux salmastricoles de la Mer Noire, Mer d'Azof et Mer Caspienne.

\subsection{Espèces endémiques pontiques-danubiennes}

Cette catégorie comprend 6 espèces appartenant à 4 genres: Theodoxus transversalis (bassins du Danube et du Dniester), $T$. danubialis (bassin des fleuves Danube, Dniester, Boug et Dniepr), Viviparus acerosus (bassin du Danube, caractéristique des secteurs moyen et inférieur), Lithoglyphus apertus (Danube moyen et inférieur, Drava et Sava), L. pigmaeus (idem), Esperiana acicularis (Danube, de l'Autriche jusqu'à la Mer Noire, ses affluents Nera et Caras, les fleuves Dniester, Boug et Dniepr) et $E$. esperi (idem).

Theodoxus transversalis et $T$. danubialis sont des espèces de néritides relictes provenant d'une lignée sarmatienne ayant, dans le pliocène, des ancêtres comme $T$. slovenicus (Brusina) et $T$. quadrifasciata (Bielz) pour la première et $T$. stefanescui (Fontannes) pour la seconde.

\subsection{Espèces endémiques pontiques-danubiennes- baltiques}

Une espèce seulement : Valvata naticina (bassins des fleuves et rivières Danube, Dniepr, Don et Oder ainsi que les marais de Pripet-Varsovie). Des coquilles fossiles du pliocène ont été trouvées dans les bassins des fleuves Dniepr et Don (pléistocène en Angleterre).

4.5. Espèces ouest et centro européennes, y compris le nord de la péninsule balkanique

Cette catégorie est réservée à Ferrissia wautieri, espèce relicte assez rare, appartenant à un genre également relicte. 


\subsection{Espèces ouest, centro et est européennes}

Seule l'aire de répartition du planorbide Anisus vorticulus correspond à cette catégorie.

\subsection{Espèces européennes}

Cinq espèces appartenant à cinq genres sont répandues partout en Europe, sauf dans les régions situées tout à fait au nord et/ou au sud : Theodoxus fluviatilis, Viviparus contectus, Potamopyrgus jenkinsi, Lithoglyphus naticoides et Anisus septemgiratus.

$T$. fluviatilis est une espèce caractéristique des provinces baltique, danubienne et pontique (y compris les bassins du Dniepr et du Don) ; dans le delta du Danube elle est remplacée par $T$. euxinus. $L$. naticoides est caractéristique du bassin du Danube et de la province pontique (y compris les bassins des fleuves et rivières Dniester, Boug, Dniepr, Donetz et Don). $A$. septemgiratus est une espèce sarmatienne ayant le centre de son aire de répartition à l'est du continent. $P$. jenkinsi est probablement importée (ou dérive d'une espèce apparentée) de Nouvelle Zélande.

\subsection{Espèçes circumméditerranéennes, ouest et est européennes.}

A cette aire de répartition correspond seulement Physa acuta.

\subsection{Espèces ouest paléarctiques}

Un seul planorbide : Segmentina nitida.

\subsection{Espèces ouest et centro paléarctiques}

Trois planorbides appartenant à trois genres : Anisus vortex (Europe et ouest de l'Asie), Hyppeutis complanatus (Europe et ouest de l'Asie jusqu'au Ienissei et au nord de l'Afrique) et Planorbarius corneus (Europe, Anatolie et Asie jusqu'aux Monts Altai).

\subsection{Espèces paléarctiques euro-sibériennes}

Six espèces appartenant à quatre genres : Valvata cristata (Europe et Sibérie jusqu'à l'Amour), V. pulchella (Europe, Sibérie, Libie), Bithynia tentaculata (Europe; Sibérie), B. leachi (Europe et Sibérie jusqu'au Kamtchatka), Acroloxus lacustris (Europe et Sibérie) et Planorbis carinatus (Europe et Sibérie jusqu'à l'Amour).

\subsection{Espèces paléarctiques}

Dix espèces appartenant à six genres (l'une de Mesogastropoda, les autres de Basommatophora) : Valvata piscinalis (introduite en Amérique du Nord), Stagnicola corvus, S. turricula, Radix auricularia, R. ovata, R. peregra, Ancylus fluviatilis, Anisus spirorbis, $A$. rotundatus et Bathyomphalus contortus.

\subsection{Espèces holarctiques}

Neuf espèces de Basommatophores appartenant à sept genres : Physa fontinalis, Lymnaea stagnalis, Stagnicola palustris, Galba truncatula, Planorbis planorbis, Gyraulus albus, G. acronicus, G. laevis et Armiger crista.

\section{Discussion}

Il résulte de ce sommaire exposé biogéographique que la faune de Gastéropodes du secteur roumain du Danube se révèle très caractéristique et d'un grand intérêt avec ses 17 espèces endémiques dont 8 ont une aire de répartition restreinte ou très restreinte. Il faut y ajouter les deux espèces à répartition européenne, Theodoxus fluviatilis et Lithoglyphus naticoides, qui ont aussi une origine sarmatienne et leur centre de répartition dans les provinces pontique et danubienne. Ainsi sur les 54 espèces du secteur roumain du Danube, 19 espèces soit $35 \%$ de la faune des Gastéropodes; sont endémiques ou ont une origine sarmatienne.

En amont du secteur roumain du Danube; plus exactement dans les sècteurs allemand, autrichien et hongrois, vivent aussi des Gastéropodes endémiques. Outre les endémiques communes avec notre secteur, il en existe d'autres ayant une aire de répartition très restreinte (cf. Frank et al., 1990). Ainsi, dans le secteur allemand se trouve l'espèce Gyraulus rossmaessleri (Auerswald) (Danube et ses systèmes lacustres). Dans le secteur autrichien on observe quatre taxons endémiques : Belgrandiella fuchsi (Boeters) (Danube et sources froides), $\boldsymbol{B}$. hartwingschuetti Reischütz (Danube et sources froides), Horatia erythropomatia kerschneri St Zimmermann (eaux souterraines du Danube, doric stygobionte) et Bythiospeum acicula geyeri (Fuchs) (Danube, ses affluents et ses systèmes lacustres, y compris leurs eaux souterraines; observé aussi dans les eaux des grottes). Dans le secteur hongrois une seule espèce est connue; Páladilhia oshanovae L. pinter (Danube à Estergom). 
Les eaux thermales des trois secteurs analysés contiennent trois endémiques : Theodoxus prevostianus (C. Pfeiffer), apparentée avec les espèces du pléistocène de climat subtropical, toujours dans des sources thermales, Malanopsis parreyssi Philippi : relicte tertiaire décrite du lac thermal Petea, près d'Oradea-Roumanie, introduite dans l'étang Malom de Budapest, et Melanoides tuberculata (O.F. Müller) : seulement dans l'étang thermal Malom de Budapest.

Dans les secteurs slovaques, aucun endémique n'a été trouvé à ce jour.

Enfin, dans les secteurs serbe et bulgare l'espèce endémique Holandiana holandri (Férussac) - syn. Amphimelania holandri C. Pfeiffer, a été mise en évidence dans le Danube et ses affluents droits : Drava, Isker, Jantra, Lom, etc. Il faut mentionner que cette espèce relicte du tertiaire se trouve aussi dans les affluents rive gauche du Danube : Nera et Caras de la Roumanie, mais jamais dans les eaux du secteur roumain de ce fleuve !

Il convient enfin d'ajouter que, dans les secteurs allemand, autrichien et hongrois du Danube, en dehors des endémiques citées, il existe encore sept espèces absentes dans le secteur roumain : Bythinella austriaca (Frauenfeld) : est-alpine-carpatique, Myxas glutinosa (O. F. Müller) : moitié nord de l'Europe et Sibérie jusqu'au lac Baikal, Stagnicola turricula (Held) sensu Falkner 1985 : ? paléarctique, avec centre de l'aire de répartition dans le bassin du Danube, Aplexa hypnorum (Linnaeus) : holarctique, Lymnaea columella (Say) : néarctique, introduite dans des bassins artificiels de Budapest, Helisoma nigricans (Say) : néarctique, introduite avec des plantes d'aquarium dans un affluent du Danube en Hongrie et $\boldsymbol{H}$. trivolvis (Say) : néarctique, introduite selon le même processus dans le Danube à Budapest.

\section{Travaux cités}

Bacescu M. 1948. - Quelques observations sur la faune benthonique du défilé roumain du Danube : son importance zoogéographique et pratique, la description d'une espèce nouvelle de Mermithidae, Pseudomermis cazanica n. sp. Ann. Sci. Univ. Jassy, $31: 240-253$.

Busnita T., Brezeanu G., Oltean M., Popescu-Marinescu V. \& Prunescu-Arion E. 1970. - Studiul hidrobiologic al Dunarii si al afluentilor sai. In Monografia zonei Portile de Fier, Ed. Acad. Rom., Bucuresti : 85-96 ; 122-134 ; 157-173.
Cure V., Popescu-Marinescu V. \& Schneider A. 1974-1975. Dinamica zoobentosului în lacul de acumulare Portile de Fier în cel de al doilea an de la formare - 1972. Trav. Stat. « Stejarul », ser. Limnologie : 141-155.

Enaceanu V. 1967. - Fauna bentonica. In Limnologia sectorului romanesc al Dunarii. Studiu monografic. Edit. Acad. Rom., Bucuresti : 287-317.

Enaceanu V. \& Brezeanu G. 1964. - Studiul biocenozelor bentonice din Dunare, sectorul Giurgiu-Cernavoda. Hidrobiologia, (Bucuresti), 5 : 51-63.

Frank C., Jungbluth J. \& Richnovsky A. 1990. - Die Mollusken der Donau vom Schwarzwald bis zum Schwarzen Mer. Akaprint, Budapest, 142 p.

Grossu A.V. 1956. - Gastropoda Prosobranchia si Opistobranchia. In Fauna R.P. Romania, Bucuresti, 3 (2) : 1-220.

Grossu A.V. 1963. - Noi contributii la cunoasterea molustelor din cursul inferior al Dunarii. Hidrobiologia, (Bucuresti), 4 : 337-359.

Grossu A.V. 1986. - Gastropoda Romaniae. vol. I. Caractere generale, istoricul si biologia gasteropodelor. Subclasa Prosobranchia si Opistobranchia. Ed. Litera, Bucuresti : 524 p.

Grossu A.V. 1987. - Gastropoda Romaniae vol. II. Subclasa Pulmonata, Ord. Basommatophora, Ord. Stylommatophora, Suprafam : Succinaea, Cochlicopacea, Pupillacea. Ed. Litera, Bucuresti : 443 p.

Grossu A.V. 1993. - Gasteropodele din Romania, compendu, Tipogr. IPCT, Bucuresti : 412 p.

Grossu A.V. \& Grossu D. 1968. - Okologische Betrachtungen über die Molluskenfauna im rumänischen Sektor des DonauFlusses und im Delta : Zuzammensetzung, Gemeinschaft, Verhältnisse, Frequenz. Arch. Hydrobiol, Suppl. 34 (3) : 168-203.

Grossu A.V. \& Negrea A. 1975. - Eine relikter Gattung der rumänischen Fauna : Ferrissia Walker 1903 (Gastropoda). Malakol. Abh. Mus. Thier., Dresda, 4 (21) : 191-196.

Grossu A.V. \& Paladian G. 1956. - Contributii la studiul molustelor din Delta si bazinul Dunarii inferioare. An. Inst. Cerc. Pisc., Bucuresti, 1 : 401-410.

Jaeckel S.G.A. 1967. - Gastropoda. In Limnofauna Europaea, Stuttgart : 89-104.

Markovskii I.M. 1955. - Fauria bezpozvonocinih nizoviev rek Ukraini uslovia ee suschcestvovania i puti ispolzovania, ciasti III : Vodoemi Kiliiskoi Delti Dunaia. Izd. Akad. Nauk. Ucr., Kiev : $279 p$.

Negrea A. \& Popescu-Marinescu V. 1992. - Gasteropodele din sectorul românesc al Dunarii gasite pîna în prezent. Hidrobiologia, (Bucuresti), $20: 75-110$.

Negrea S. \& Negrea A. 1975. - Ecologia populatiilor de Cladoceri si Gasteropode din zona inundabila a Dunarii. Ed. Acad. Rom., Bucuresti : 232 p.

Popescu E. \& Prunescu-Arion E. 1961. - Contributii la studiul faunei bentonice din Dunare, în regiunea cataractelor $(\mathrm{km}$ 1042-955). Stud. cerc. biol., ser. Biol. anim., 13 (2) : 237-256.

Popescu L. \& Munteanu I. 1962. - Contributii la cunoasterea componentei si repartitiei bentosului în Dunare (sectorul Ceatal Izmail - Sf. Gheorghe). Bul. Inst. Cerc. Pisc., 21 (4) : 67-79.

Popescu V. 1963. - Studiul hidrobiologic al bratului Sulina. Hidrobiologia, (Bucuresti), 4 : 215-255. 
Popescu-Gorj A. \& Costea E. 1961. - Cercetari hidrobiologice si piscicole în baltile Oltinei (lunca inundabila a Dunarii). Hidrobiologia, (Bucuresti), 2 : 29-125.

Popescu-Gorj A., Popescu E. \& Georgescu I. 1957. - Studiul hidrobiologic-piscicol al complexului Pardina (Delta Dunarii). An. Inst. Cerc. Pisc. ser.nov. 1-4 : 51-104.

Popescu-Marinescu V. 1973. - Repartitia si componenta organismelor animale din biocenozele bentonice si fitofile, în bratele Dunarii si Delta Dunarii. Peuce (Tulcea) St. comunic. st. nat., 3 : 185-209.

Popescu-Marinescu V. 1992. - Structura zoocenozelor bentonice din Dunare, în sectorul românesc, în perioada 1971-1986. Hidrobiologia, (Bucuresti), 20 : 111-134.
Popescu-Marinescu V., Elian-Talau L., Stoica A. \& Izvoranu V. 1980. - Cercetari hidrobiologice asupra Dunarii si Oltului în zona de confluenta. Hidrobiologia, (Bucuresti), 16 : 203-213.

Prunescu-Arion E., Elian-Talau L. \& Baltac M. 1965. - Influenta viiturilor Dunarii asupra chimismului si biologiei gîrlei Saltava si canalului Filipoiu din lunca inundabila. Hidrobiologia, (Bucuresti), $6:$ 151-167.

Russev B. 1967. - Das zoobenthos der Donau. In Limnologie der Donau, Stuttgart, 3 : 242-271.

Starobogatov I.I. 1970. - Fauna Molliuskov i zoogeograficeskoe raionirovanie kontinentalnih vodoemov zemnogro Schara. Izd. Nauka, Leningrad : 320 p.

Zilch A. \& Jaeckel S.G.A. 1962. - Die Weichtiere (Mollusca). In Die Tierwelt Mitteleuropas, Ergänzung, 2 (1) : 1-249. 